\title{
Effects of oxygen content and thermal annealing on sputtered a-IGZO Schottky diodes
}

\author{
Veronika Ulianova \\ Empa - Swiss Federal Laboratories for \\ Materials Science and Technology \\ Dübendorf, Switzerland \\ veronika.ulianova@empa.ch \\ Ivan Shorubalko \\ Empa - Swiss Federal Laboratories for Materials Science and \\ Technology \\ Dübendorf, Switzerland \\ ivan.shorubalko@empa.ch
}

Sami Bolat

Empa - Swiss Federal Laboratories for Materials Science and Technology

Dübendorf, Switzerland sami.bolat@empa.ch
Galo Torres Sevilla

Empa - Swiss Federal Laboratories for Materials Science and Technology

Dübendorf, Switzerland

galo.torres-sevilla@empa.ch

Yaroslav Romanyuk

Empa - Swiss Federal Laboratories for Materials Science and Technology

Dübendorf, Switzerland

yaroslav.romanyuk@empa.ch

\begin{abstract}
Effects of oxygen content during sputtering of amorphous In-Ga-Zn-O (a-IGZO) and post-deposition thermal annealing on the properties of vertical thin-film Schottky diodes were investigated. Increase of the oxygen content in the $\mathrm{Ar} / \mathrm{O}_{2}$ mixture from 0 to $20 \%$ led to lower current densities. At the same time higher oxygen content enhanced the $I_{0 n} / I_{\text {off }}$ ratio of the Schottky diodes without post-treatment. The currentvoltage characteristics exhibited a shift of the current minimum point depending on the bias sweep direction for the devices fabricated at $11.76 \%$ and $20 \%$ of oxygen. The postdeposition thermal annealing in air was found to improve the $I_{\text {on }} / I_{\text {off }}$ ratio with an optimum annealing temperature of $200^{\circ} \mathrm{C}$ although the effect depended on the initial oxygen content. Ideality factor and barrier height were calculated for all fabricated devices. Schottky diodes on glass based on IGZO sputtered at $3.23-6.25 \%$ of oxygen with $I_{\text {on }} / I_{\text {off }}$ ratio of $2.6 \cdot 10^{6}$ $1.6 \cdot 10^{7}$ at $\pm 1 \mathrm{~V}$, ideality factor of $1.3-1.5$, and barrier height of $0.62-0.67 \mathrm{eV}$ were obtained after annealing at $200^{\circ} \mathrm{C}$.
\end{abstract}

Keywords-Schottky diode, amorphous IGZO, sputtering, oxygen content, thermal annealing

\section{INTRODUCTION}

Amorphous In-Ga-Zn-O (IGZO) is an established semiconductor material for the next generation of electronic devices due to its optical transparency, high mobility, low temperature processability, and easy structuring because of amorphous nature. Initially, IGZO was applied as a channel material of thin-film transistors [1], later, Schottky diodes based a-IGZO have been demonstrated for novel applications such as ultra-high frequency energy harvesting system [2], flexible electronics on paper [3], and transparent electronics [4].

In the quaternary system of IGZO, $\mathrm{In}^{3+}$ ions can enhance the mobility by providing a primary conduction pathway, $\mathrm{Ga}^{3+}$ ions act as a carrier suppressor due to Ga's strong oxygen affinity, $\mathrm{Zn}^{2+}$ ions introduce cation size variation in the structure, which helps to stabilize the amorphous phase and $\mathrm{O}^{2-}$ also influences the electronic performance of the oxide film, because oxygen vacancies are the major source of free electrons [5]. For example, the fabricated Schottky diodes based on IGZO with a composition of In:Ga: $\mathrm{Zn}=1.0: 0.8: 0.3$ exhibited a rectification ratio of $8.3 \cdot 10^{6}$ [6]. Therefore elemental ratio has a significant effect on the final device performance. Particularly in case of oxide semiconductors all of the manufacturing parameters such as pressure, temperature, atmosphere, postand pre-deposition treatments lead to the modification of oxygen concentration in the thin film itself and at the device interfaces, and this way effect on electrical properties of the final device.

Schottky diodes with a rectification ratio up to $10^{8}$ and ideality factor of 1.22 have been developed at $20 \%$ of oxygen content during the sputtering and post-annealing at $200^{\circ} \mathrm{C}$ [7]. Moreover in that research bottom Pd electrode was treated with oxygen plasma before IGZO sputtering to increase the oxygen level in the IGZO and at the Schottky electrode, because this reduces the IGZO subgap states and Fermi level pinning [7]. To reduce the effect of the surface defects concentration UV-ozone treatment of $\mathrm{Pd}$ and $\mathrm{Au}$ electrodes were applied and diodes with rectification ratio of $10^{7}$ were developed [8]. The device with barrier height of $0.92 \mathrm{eV}$ and ideality factor of 1.36 were obtained after introduction of $\mathrm{Ar} / \mathrm{O}_{2}$ gases during the deposition of the $\mathrm{Pt}$ layer to reduce the oxygen deficiency at the Schottky interface [9]. Gradual doping of oxygen from $0 \%$ to $37.5 \%$ along the vertical profile of the a-IGZO layer allowed achieving the transparent conducting oxide/a-IGZO Schottky junction with rectification ratio of $10^{3}$ [4]. Effects of substrate and anode metal annealing on IGZO Schottky diodes were studied and it was found that annealing of $\mathrm{Si} / \mathrm{SiO}_{2}$ substrate improved the performance of the devices [10].

Thus, different technological approaches and regimes are intensively investigated to improve the performance of aIGZO Schottky diodes. The most part of studies have been done for $20 \%$ of oxygen content during sputtering and thermal annealing at $150-200^{\circ} \mathrm{C}$ for $\mathrm{Pd} / \mathrm{IGZO}$ Schottky contact interface; but it is not clear if these oxygen content and annealing temperature are optimum, and if they can be applied also for other high work function metal contacts.

In this work we investigated effects of oxygen content in a gas mixture during IGZO sputtering, post-deposition thermal annealing and combination of both approaches on rectification ratio, ideality factor and Schottky barrier height of the vertical thin-film Pt-IGZO-Cu Schottky diode structure.

\section{MATERIALS AND Methods}

The vertical device configuration was chosen for the research because of higher current density and better scalability compared to lateral devices (Fig. 1, a). Soda lime glass was used as a substrate after cleaning in acetic acid and deionized water in an ultrasonic bath at $80^{\circ} \mathrm{C}$ for $40 \mathrm{~min}$.

Since electron affinity of IGZO with an atomic ratio of $\ln : G a: Z n=1: 1: 1$ equals to $4.16 \mathrm{eV}$ [11], the metal with work

This document is the accepted manuscript version of the following article:

Ulianova, V., Bolat, S., Torres Sevilla, G., Shorubalko, I., \& Romanyuk, Y. (2019). Effects of oxygen content and thermal annealing on sputtered a-IGZO Schottky diodes. In 2019 IEEE 39th International Conference on electronics and nanotechnology (ELNANO). Conference proceedings (pp. 88-91). 
function exceeding $5 \mathrm{eV}$ is needed to achieve a high potential barrier. The bottom electrode was deposited by radio frequency (RF) sputtering of $\mathrm{Pt}$ on $\mathrm{Ti}$ adhesive layer at a pressure of $0.28 \mathrm{~Pa}$ and a power density of $2.96 \mathrm{~W} \cdot \mathrm{cm}^{-2}$. Total thickness of the bottom electrode was $100 \mathrm{~nm}$. Amorphous IGZO layer $(60-70 \mathrm{~nm})$ was deposited by RF magnetron sputtering from an IGZO target (composition $\ln : \mathrm{Ga}: \mathrm{Zn}=1: 1: 1)$ in an $\mathrm{Ar} / \mathrm{O}_{2}$ atmosphere with oxygen concentration of $0-20 \%$ at a pressure of $0.6 \mathrm{~Pa}$ at room temperature and a power density of about $1 \mathrm{~W} \cdot \mathrm{cm}^{-2}$. Before deposition of the top contact the structures were annealed at 200,250 or $300^{\circ} \mathrm{C}$ for $1 \mathrm{~h}$ on a hot plate in air to investigate the effect of thermal annealing on properties of the Schottky diodes.

The ohmic $\mathrm{Cu}$ top contact was formed by evaporation through a metal mask under high vacuum $\left(<4 \cdot 10^{-4} \mathrm{~Pa}\right)$. The area of the contact was $200 \times 200 \mu \mathrm{m}$ and the thickness of the layer was $70 \mathrm{~nm}$.

The top-view microscope photograph of the fabricated devices is shown in Fig. 1, b.

The current-voltage characteristics of the diodes were measured in air at room temperature in dark without encapsulation using Keithley 4200 characterization system; electrodes were contacted using a probe station Karl Suss PM8.

\section{RESULTS AND DISCUSSION}

The typical current density - voltage characteristics of Schottky diodes fabricated at $0-20 \%$ of oxygen content during IGZO RF sputtering and after thermal annealing are presented in Fig. 2. By changing the oxygen content in the gas mixture during the IGZO sputtering the electron density is changed, that effects on current density of the devices. Thus, with increase of oxygen content in the $\mathrm{Ar} / \mathrm{O}_{2}$ mixture from 0 to $20 \%$, current density lowers from 250 to $0.03 \mathrm{~A} / \mathrm{cm}^{2}$ at $1 \mathrm{~V}$ for the samples without thermal treatment (Fig. 2, a). The decrease in carrier concentration at larger $\mathrm{O}_{2}$ content was observed for top-gate transparent thin-film transistors based on IGZO [12]. Current densities at $1 \mathrm{~V}$ start to increase after thermal annealing at $200^{\circ} \mathrm{C}$ for the samples deposited in the presence of oxygen (Fig. 2, b-d). This effect can be explained by changing of the free charge density by changing the concentration of oxygen during the annealing.

The current rectification ratio $\left(\mathrm{I}_{\mathrm{on}} / \mathrm{I}_{\mathrm{off}}\right)$ of the fabricated Schottky diodes as well as an ideality factor and Schottky barrier height were calculated from IV measurement in forward voltage sweep (from -1 to $+1 \mathrm{~V}$ ). Thus, $\mathrm{I}_{\text {on }} / \mathrm{I}_{\text {off }}$ of Schottky diodes sputtered at $0-11.76 \%$ of oxygen increases from 1 to $6.6 \cdot 10^{7}$ without post-treatment as shown in Fig. 3, suggesting that oxygen concentration during sputtering also effects the Pt/IGZO junction characteristics.

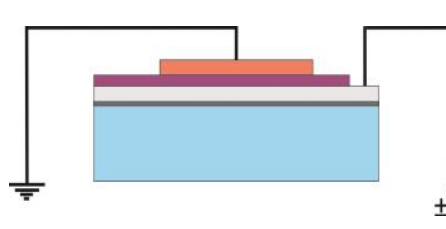

a)
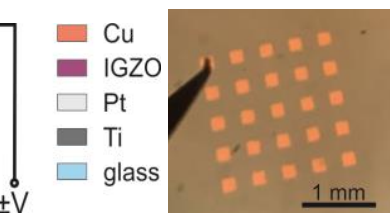

b)
Fig. 1. Cross-sectional schematic (a) and top-view image (b) of the IGZO diodes.
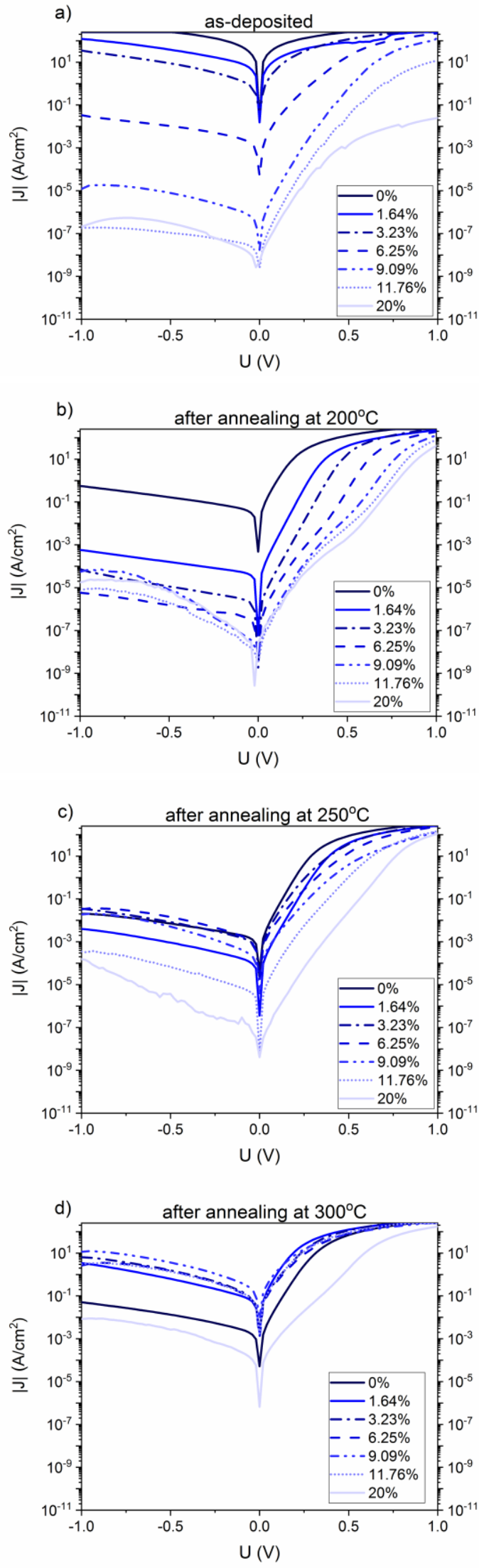

Fig. 2. J-V dependencies of Schottky diodes based on the Pt-IGZO-Cu structure with variable oxygen content of the $\mathrm{Ar} / \mathrm{O}_{2}$ gases during IGZO RF sputtering: (a) - without thermal treatment, (b) - after annealing at $200^{\circ} \mathrm{C}$, (c) - after annealing at $250^{\circ} \mathrm{C}$, (d)- after annealing at $300^{\circ} \mathrm{C}$. 


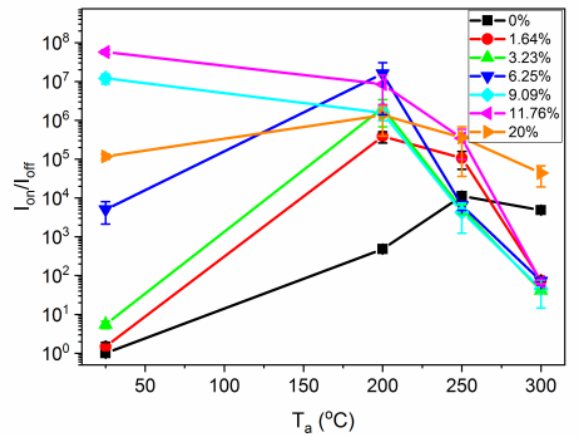

Fig. 3. Dependence of $I_{o n} / I_{o f f}$ at $\pm 1 V$ on annealing temperature $T_{a}$ for PtIGZO-Cu structures with variable oxygen content of the $\mathrm{Ar} / \mathrm{O}_{2}$ gases during IGZO RF sputtering.

The effect of annealing temperature on $\mathrm{I}_{\mathrm{on}} / \mathrm{I}_{\mathrm{off}}$ ratio depends on the initial oxygen content. The improvement of rectification ratio (up to $1.6 \cdot 10^{7}$ at $\pm 1 \mathrm{~V}$ for $6.25 \%$ of oxygen) is achieved for the samples fabricated at $0,1.64$, $3.23,6.25$ and $20 \%$ of oxygen after annealing at $200^{\circ} \mathrm{C}$ (Fig. 2, b and Fig. 3). In comparison, the rectification ratio of $10^{7}$ at $\pm 1 \mathrm{~V}$ was achieved after annealing at $200^{\circ} \mathrm{C}$ of the $\mathrm{Pd}-$ IGZO-Mo structure with IGZO layer sputtered at $20 \%$ of oxygen [2]. The rectification ratio increases from 1 after deposition to maximum value of $10^{4}$ after annealing at $250^{\circ} \mathrm{C}$ only for samples fabricated in $\mathrm{Ar}$ atmosphere, while decreases for all others samples after annealing at $250^{\circ} \mathrm{C}$ (Fig. 3). The drop of rectification ratio is observed for all samples after annealing at $300^{\circ} \mathrm{C}$ (Fig. 2, d and Fig. 3). The deterioration of parameters was also observed after annealing at $150^{\circ} \mathrm{C}$ of the $\mathrm{Pd} / \mathrm{IGZO}, \mathrm{Pt} / \mathrm{IGZO}$ and $\mathrm{Au} / \mathrm{IGZO}$ structures with IGZO deposited in $20 \%$ of oxygen [8].

The current density - voltage characteristics of the samples deposited at $11.75 \%$ and $20 \%$ of oxygen exhibit hysteresis and a shift of the current minimum point depending on the bias sweep direction (Fig. 4). From the model of thermionic emission, the shift of current minimum to positive or negative voltages depending on sweep direction might be caused by charging and discharging of trap states [11]. After annealing the effect of the bias sweep direction was lowered, but not eliminated.

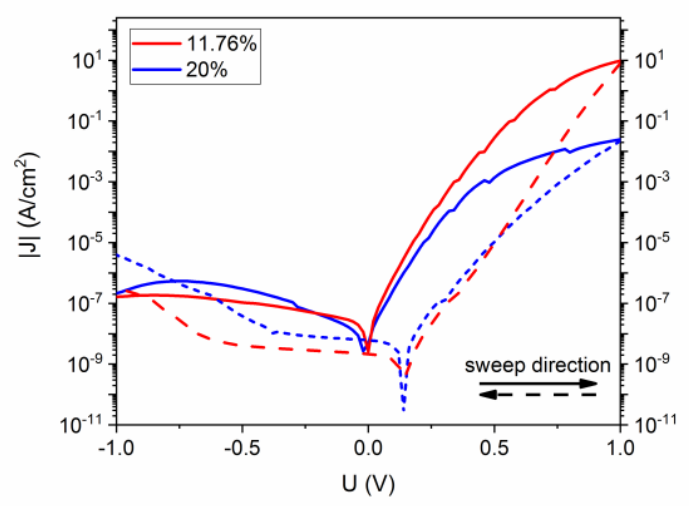

Fig. 4. J-V characteristics of the diodes based on the Pt-IGZO-Cu structure with $11.76 \%$ and $20 \%$ of oxygen in the $\mathrm{Ar} / \mathrm{O}_{2}$ mixture during IGZO RF sputtering.
The current transport through a Schottky junction is described by the thermionic emission of majority carriers over the junction barrier [13].The ideality factor $n$ is included in the I-V relationship to take into account nonideal diode behaviors:

$$
I=I_{s}\left[\exp \left(q V_{D} / n k T\right)-1\right]
$$

where $q$ - is the charge of the electron, $V_{D}$ - applied voltage $V_{D}=V-I R$ ( $V$ is the total voltage drop across the series combination of the diode and the resistor $R$ through which the current $I$ flows), $k$ - the Boltzmann constant and $T$ - the absolute temperature. $I_{s}$ is expressed by:

$$
I_{s}=A_{e f f} A^{*} T^{2} \exp \left(-q \phi_{B} / k T\right)
$$

where $A_{\text {eff }}$ - is the effective area of the diode $(200 \times 200 \mu \mathrm{m})$, $A^{*}$ - is the effective Richardson constant, which for IGZO has a theoretical value of $41 \mathrm{~A} \cdot \mathrm{cm}^{-2} \mathrm{~K}^{-2}$ [14] and $\phi_{B}-$ is the Schottky barrier height of the diode. After rewriting of Equation (1) in term of current density $J$ :

$$
V=R A_{e f f} J+n \phi_{B}+(n / \beta) \ln \left(J / A^{*} T^{2}\right),
$$

where $\beta=q / k T$.

After differentiating of Equation (3), $n / \beta$ can be extracted as the $y$-axis intercept from the plot of $d(V) / d(\ln J)$. To evaluate $\phi_{B}$ a function $H(J)$ was defined similar to [13]:

$$
H(J)=V-(n / \beta) \ln \left(J / A^{*} T^{2}\right) .
$$

From a plot of $H(J)$ :

$$
H(J)=R A_{e f f} J+n \phi_{B},
$$

$n \phi_{B}$ was extracted as y-axis intercept.

An ideality factor and Schottky barrier height extracted from the forward current-voltage characteristics of the fabricated devices are shown in Fig. 5 and Fig. 6, respectively. To calculate standard deviation of the parameters (Fig. 3, 5 and 6) characteristics of at least 4 devices were used.

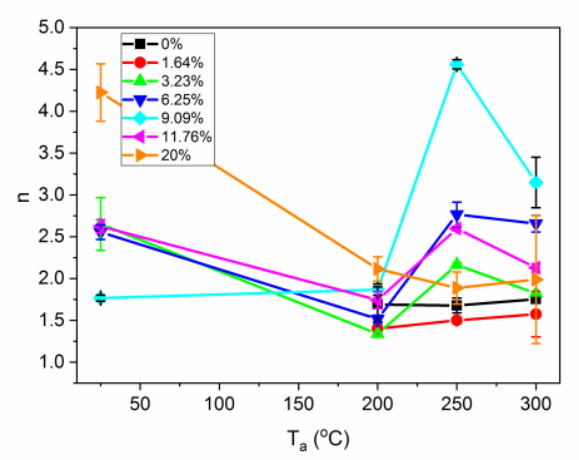

Fig. 5. Dependence of ideality factor on annealing temperature $\mathrm{T}_{\mathrm{a}}$ for PtIGZO-Cu structures with variable oxygen content of the $\mathrm{Ar} / \mathrm{O}_{2}$ gases during IGZO RF sputtering (calculated from I-V measurements). 


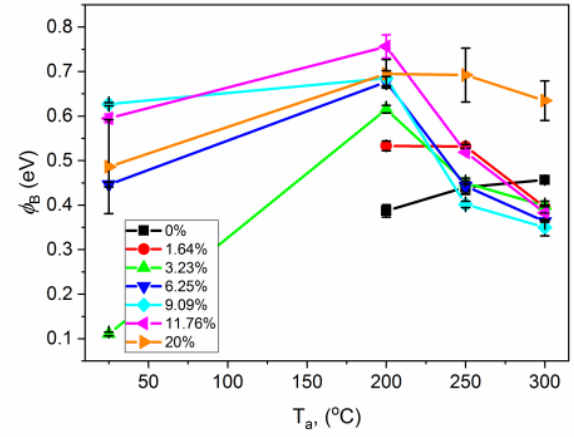

Fig. 6. Dependence of barrier height on annealing temperature $\mathrm{T}_{\mathrm{a}}$ for PtIGZO-Cu structures with variable oxygen content of the $\mathrm{Ar} / \mathrm{O}_{2}$ gases during IGZO RF sputtering (calculated from IV measurements).

As it is shown in Fig. 5, the annealing temperature has almost the same effect on the ideality factor for the diodes based on IGZO sputtered as well as without oxygen and in the presence of oxygen up to $11.76 \%$ with the lowest values of $1.33-1.86$ after annealing at $200^{\circ} \mathrm{C}$, but for the devises fabricated at $20 \%$ of oxygen the ideality factor is improved to the best value of 1.6 at $250^{\circ} \mathrm{C}$. Subsequent increase of annealing temperature to $300^{\circ} \mathrm{C}$ led to characteristics degradation of the devices fabricated in presence of oxygen during IGZO sputtering.

Schottky barrier height of $0.6-0.75 \mathrm{eV}$ is obtained for the Pt-IGZO-Cu Schottky diodes with IGZO sputtered in the presence of oxygen after annealing at $200^{\circ} \mathrm{C}$. The decrease of the barrier height is observed with increase of annealing temperature. In comparison, the extracted barrier heights for Pt-IGZO-Al Schottky diodes based on IGZO sputtered in 3\% of oxygen without any thermal annealing process were $0.79 \mathrm{eV}, 0.73 \mathrm{eV}$ and $0.65 \mathrm{eV}$ for the diodes with $100 \mathrm{~nm}$, $80 \mathrm{~nm}$, and 50nm IGZO layer [15].

\section{CONCLUSIONS}

Vertical thin-film Pt-IGZO-Cu Schottky diodes were fabricated. By changing the oxygen content in the $\mathrm{Ar} / \mathrm{O}_{2}$ mixture during the IGZO sputtering the electron density is changed, that effects on the current density of the devices as well as on rectification ratio, ideality factor and Schottky barrier height. The highest rectification ratio of $6.6 \cdot 10^{7}$ for Pt-IGZO Schottky diodes without post-treatment was achieved after increasing oxygen content to $11.75 \%$, at the same time the ideality factor for this devices was calculated as 2.63 and Schottky barrier height - as $0.6 \mathrm{eV}$.

The effect of annealing temperature on $\mathrm{I}_{\text {on }} / \mathrm{I}_{\text {off }}$ ratio, ideality factor and Schottky barrier height depended on the initial oxygen content. The post-deposition thermal annealing at $200^{\circ} \mathrm{C}$ in air was found to improve the $\mathrm{I}_{\text {on }} / \mathrm{I}_{\text {off }}$ ratio at $\pm 1 \mathrm{~V}$ to $2.610^{6}-1.610^{7}$, as well as to lower ideality factor of 1.3 - 1.5 and Schottky barrier height $0.62-0.67 \mathrm{eV}$ for the devices fabricated at $3.23-6.25 \%$ of oxygen. Thus, obtained results demonstrated effects of oxygen concentration during RF sputtering and post-deposition thermal annealing on the properties of $\mathrm{Pt} / \mathrm{IGZO}$ Schottky diodes.

\section{ACKNOWLEDGMENT}

The EMPAPOSTDOCS-II programme has received funding from the European Union's Horizon 2020 research and innovation programme under the Marie SkłodowskaCurie grant agreement number 754364 .

\section{REFERENCES}

[1] T. Kamiya, K. Nomura, and H. Hosono, "Present status of amorphous In-Ga-Zn-O thin-film transistors," Sci. Technol. Adv. Mater., vol. 11, no. 4, p. 044305, Feb. 2010.

[2] A. Chasin et al., "Integrated UHF a-IGZO energy harvester for passive RFID tags," in 2013 IEEE International Electron Devices Meeting, 2013, pp. 11.3.1-11.3.4.

[3] J. Kaczmarski, M. A. Borysiewicz, K. Piskorski, M. Wzorek, M. Kozubal, and Eliana Kamińska, "Flexible IGZO Schottky diodes on paper," Semicond. Sci. Technol., vol. 33, no. 1, p. 015010, 2018.

[4] S. Ho, H. Yu, and F. So, "Transparent indium-tin oxide/indiumgallium-zinc oxide Schottky diodes formed by gradient oxygen doping," Appl. Phys. Lett., vol. 111, no. 21, p. 212103, Nov. 2017.

[5] J. K. Jeong, J. H. Jeong, H. W. Yang, J.-S. Park, Y.-G. Mo, and H. D. Kim, "High performance thin film transistors with cosputtered amorphous indium gallium zinc oxide channel," Appl. Phys. Lett., vol. 91, no. 11, p. 113505, Sep. 2007.

[6] J.-W. Kim, T.-J. Jung, and S.-M. Yoon, "Device characteristics of Schottky barrier diodes using In-Ga-Zn-O semiconductor thin films with different atomic ratios," J. Alloys Compd., vol. 771, pp. 658663, Jan. 2019.

[7] A. Chasin et al., "Gigahertz Operation of a-IGZO Schottky Diodes," IEEE Trans. Electron Devices, vol. 60, no. 10, pp. 3407-3412, Oct. 2013.

[8] A. Chasin et al., "High-performance a-In-Ga-Zn-O Schottky diode with oxygen-treated metal contacts," Appl. Phys. Lett., vol. 101, no. 11, p. 113505, Sep. 2012.

[9] J. Zhang, Q. Xin, and A. Song, "High performance Schottky diodes based on indium-gallium-zinc-oxide," J. Vac. Sci. Technol. A, vol. 34, no. 4, p. 04C101, Apr. 2016.

[10] L. Du et al., "Effects of substrate and anode metal annealing on InGaZnO Schottky diodes," Appl. Phys. Lett., vol. 110, no. 1, p. 011602, Jan. 2017.

[11] T.-C. Fung et al., "Two-dimensional numerical simulation of radio frequency sputter amorphous $\mathrm{In}-\mathrm{Ga}-\mathrm{Zn}-\mathrm{O}$ thin-film transistors," $J$. Appl. Phys., vol. 106, no. 8, p. 084511, Oct. 2009.

[12] H.-H. Hsieh, C.-H. Wu, C.-W. Chien, C.-K. Chen, C.-S. Yang, and C.-C. Wu, "Influence of channel-deposition conditions and gate insulators on performance and stability of top-gate IGZO transparent thin-film transistors," J. Soc. Inf. Disp., vol. 18, no. 10, pp. 796-801, 2010.

[13] S. K. Cheung and N. W. Cheung, "Extraction of Schottky diode parameters from forward current-voltage characteristics," Appl. Phys. Lett., vol. 49, no. 2, pp. 85-87, Jul. 1986.

[14] A. Takagi et al., "Carrier transport and electronic structure in amorphous oxide semiconductor, a-InGaZnO4," Thin Solid Films, vol. 486, no. 1, pp. 38-41, Aug. 2005.

[15] J. Zhang, Y. Li, B. Zhang, H. Wang, Q. Xin, and A. Song, "Flexible indium-gallium-zinc-oxide Schottky diode operating beyond 2.45 GHz,” Nat. Commun., vol. 6, p. 7561, Jul. 2015. 